\title{
COLD ANTIBODY-MEDIATED AUTOIMMUNE HEMOLYTIC ANEMIA IN A PATIENT WITH OVERLAP SYNDROME OF SYSTEMIC LUPUS ERYTHEMATOSUS WITH SJOGREN'S SYNDROME
}

\author{
INDIRA BAIRY ${ }^{1}$, ANUPAMBERWAL ${ }^{2}$, CHARANTHEJREDDY $\mathrm{V}^{3}$, MUKHYAPRANAPRABHU ${ }^{3}$
}

\begin{abstract}
${ }^{1}$ Department of Microbiology, Melaka Manipal Medical College, Manipal Campus, Manipal Academy of Higher Education, Manipal, Karnataka, India. ${ }^{2}$ Department of Microbiology, Kasturba Medical College, Manipal Academy of Higher Education, Manipal, Karnataka, India. ${ }^{3}$ Department of Medicine, Kasturba Medical College, Manipal Academy of Higher Education, Manipal, Karnataka, India. Email: ???
\end{abstract}

Received: 21 July 2018, Revised and Accepted: 25 October 2018

ABSTRACT

Systemic lupus erythematosus (SLE) is a systemic autoimmune disorder commonly seen in females characterized by multisystem inflammation with the production of an array of antibodies. Hematological disturbances are common in SLE specifically autoimmune hemolytic anemia (AIHA) which results from the development of autoantibodies directed against antigens on the surface of patient's own red blood cells. Here, we present a rare case of a 50-year-old female patient presenting with cold antibody-mediated AIHA.

Keywords: Autoimmune, Rituximab, Anemia.

(c) 2019 The Authors. Published by Innovare Academic Sciences Pvt Ltd. This is an open access article under the CC BY license (http://creativecommons. org/licenses/by/4. 0/) DOI: http://dx.doi.org/10.22159/ajpcr.2019.v12i2.28644

\section{INTRODUCTION}

Systemic lupus erythematosus (SLE) is a chronic inflammatory disease commonly affecting women. SLE is a systemic autoimmune disorder characterized by multisystem inflammation with the production of an array of antibodies. Serum nuclear antibodies (antinuclear antibody [ANA]) are found in nearly all individuals with active SLE [1]. Autoimmune hemolytic anemia (AIHA) in SLE is usually mediated by warm immunoglobulin G anti-erythrocyte antibodies [2]. However, here, we report a female patient who was a known case of SLE, presented with cold antibody-mediated AIHA.

\section{CASE REPORT}

A 50-year-old woman presented to the department of medicine with dysphagia and passage of dark stools for 1 week. Dysphagia started 1 year back, and she was diagnosed as SLE/overlap syndrome (SLE Sjogren's syndrome and Raynaud's phenomenon). She was started on steroids, after which the symptoms subsided for 2-3 months. Dysphagia started again for 1 week which was increasing gradually. The patient complained of the passage of loose stools for 7 days, dark, sticky, mucoid, and scanty in amount associated with painful defecation. The patient gives a history of passing blood in urine which increased on exposure to cold. She was taken to a local hospital with complaints of fatigue and dysphagia and was referred to our tertiary care hospital.

There was no history of autoimmune disease in other family members.

On examination, the patient was conscious and well oriented. Pallor was present, and there were no icterus, no clubbing, and no lymphadenopathy. Cyanosis of the fingers of the left hand was present. Her blood pressure was $110 / 70 \mathrm{mmHg}$, pulse 84 beats/min, respiratory rate $18 / \mathrm{min}$, temperature $98^{\circ} \mathrm{F}$, and $\mathrm{SpO}_{2} 98 \%$ on room air. Her chest was clear, and the abdomen was soft, non-distended, and non-tender with no organomegaly. Cardiovascular system examination was normal.

\section{Investigations}

Her initial laboratory workup revealed peripheral smear showing spherocytosis. Serum bilirubin: Total $-1.41 \mathrm{mg} / \mathrm{dl}$, direct $-0.09 \mathrm{mg} / \mathrm{dl}$, indirect $-1.32 \mathrm{mg} / \mathrm{dl}$, alkaline phosphatase - $73 \mathrm{IU} / \mathrm{L}$ (normal<130 IU/L), alanine aminotransferase - $17 \mathrm{IU} / \mathrm{L}$ (normal $<40 \mathrm{IU} / \mathrm{L}$ ), aspartate aminotransferase-63IU/L(normal<40IU/L), erythrocytesedimentation rate $-60 \mathrm{~mm} / \mathrm{h}$, serum albumin $-4.21 \mathrm{~g} \%$, hemoglobin $-2.4 \mathrm{~g} / \mathrm{dl}$, total white blood cell - 8800/ $\mathrm{mm}^{3}$, differential count - PMN 79.9/L 12.6/E 2.2/M 4.7\%, and platelet count - 309,000/ $\mathrm{mm}^{3}$. Peripheral smear showed extensive red blood cell (RBC) clumping as shown in Fig. 1.

ANA immunofluorescence on human epithelial type 2 and rat liver cells showed nucleus granular pattern $(+++)$ with a titer of $>1: 320$. The ANA profile showed Ro-52 (+++), dsDNA +++, Sm (+), and SS-B (+). Antineutrophil cytoplasmic antibody immunofluorescence was negative.

The laboratory workup for infections including viral hepatitis, tuberculosis, and HIV was negative. In view of dysphagia, ultrasonography was done, which revealed lax esophagogastric junction with duodenogastric reflux and suggested to do manometry. Manometry revealed abnormal contractility. Workup for hemolysis showed cold antibody positive. The case was diagnosed as SLE overlap syndrome (SLE with Sjogren's syndrome and Raynaud's phenomenon) with severe anemia mediated by cold autoantibodies and esophageal dysmotility.

\section{Treatment}

The patient was on methotrexate $15 \mathrm{mg} /$ week for joint pain for 1 year. Initially, she was treated with prednisolone $1 \mathrm{mg} / \mathrm{kg}$, but the patient continued to have hemolysis, and there was no improvement. Then, after review of literature, rituximab was considered as an option. The patient was treated for SLE-related intravascular hemolysis. Three units of packed RBC (PRBC) transfusions were given under warmer in view of dropping hemoglobin. However, treatment with steroids proved ineffective and the patient required further blood transfusions for symptomatic anemia. Rituximab was initiated resulting in significant clinical improvement. Her hemoglobin improved from $3.8 \mathrm{~g} / \mathrm{dl}$ to $12 \mathrm{~g} / \mathrm{dl}$. The patient was subsequently discharged from the hospital.

\section{Outcome and follow-up}

The patient was continued on rituximab as outpatient and did not require any further blood transfusions. Her follow-up hematocrit levels remained stable. Over 1 month follow-up course, she showed remarkable improvement in her symptomatology and other clinical parameters including normalization of her hemoglobin levels. 


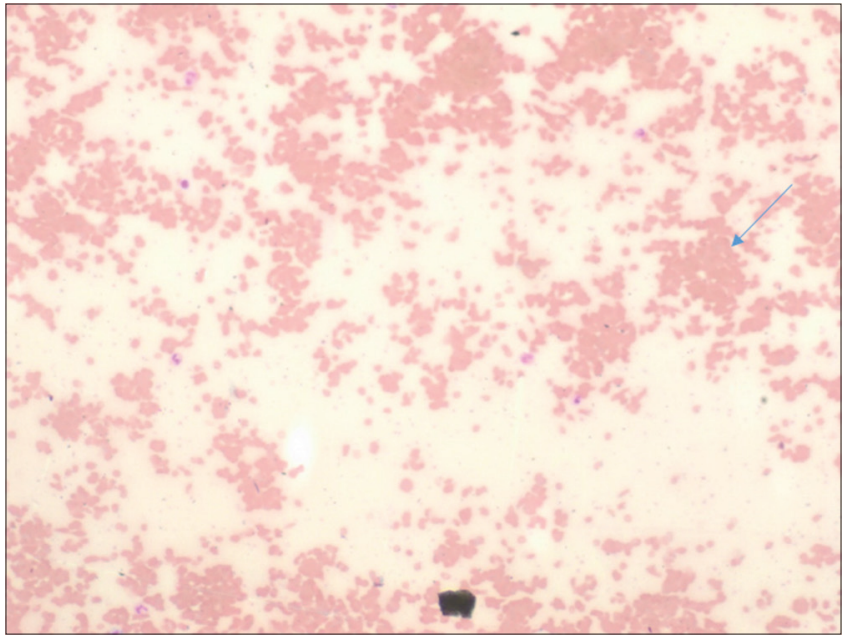

Fig. 1: Peripheral blood smear showing extensive red blood cell clumping at $\times \mathbf{1 0 0}$

\section{DISCUSSION}

Most patients with SLE develop hematological abnormalities during their natural course of the disease. Anemia is found in about $50 \%$ of patients, and the most common is anemia of chronic disease, followed by iron deficiency anemia, AIHA, drug-related myelotoxicity, and anemia of chronic kidney disease [2].

Antibody-mediated RBC damage is one of the most common mechanisms of anemia in SLE, and it is almost always mediated by warm antibodies. Acquired deficiency of RBC CD55 and/or CD59 is associated with AIHA in SLE. Similar mechanism can cause autoimmune thrombocytopenia and lymphopenia. Immune-mediated marrow failure is another mechanism for anemia/pancytopenia. First line of treatment for AIHA is prednisone ( $1 \mathrm{mg} / \mathrm{kg} /$ day). Approximately $80 \%$ of patients will show a dramatic response after 3 weeks, or patients requiring above $15 \mathrm{mg} /$ day for maintenance are absolute candidates for second-line drugs. In idiopathic AIHA, only proven next line treatment with shortterm efficacy is rituximab and splenectomy.

Although extremely rare, cold antibody AIHA in SLE has been reported $[3,4]$. Binding of antibodies to RBCs activates the classical pathway of the complement system leading to the formation of membrane attack complex and intravascular hemolysis. On the other hand, if classic pathway is ineffective, RBCs are opsonized with complement proteins (particularly, C3b and C4b) which enhance phagocytosis in the liver and spleen, presenting as extravascular hemolysis. In the present case, the hemoglobin level was low, suggesting intravascular hemolysis. The peripheral blood smear confirmed the diagnosis.

Rituximab has been shown to be very efficacious in the past for AIHA with SLE refractory to conventional therapy [5]. Rituximab is also known to maintain long-term remission rate in patients with primary cold agglutinin AIHA [6,7] and showed rituximab to be extremely effective and safe therapeutic alternative in patients with intractable and severe AIHA. We used rituximab as our second-line agent. To the best of our knowledge, this is, perhaps, the first case report of CA-AIHA in a patient with overlap syndrome of SLE and Sjogren's syndrome.

\section{AUTHORS' CONTRIBUTIONS}

Dr. Indira Bairy - Edited the manuscript Dr. Anupam Berwal - Prepared the manuscript Dr. Charan - Did workup on the case

Dr. Mukhyapranaprabhu - Managed the case and also reviewed the manuscript.

\section{REFERENCES}

1. Packman $\mathrm{CH}$. The clinical pictures of autoimmune hemolytic anemia. Transfus Med Hemother 2015;42:317-24.

2. Giannouli S, Voulgarelis M, Ziakas PD, Tzioufas AG. Anaemia in systemic lupus erythematosus: From pathophysiology to clinical assessment. Ann Rheum Dis 2006;65:144-8.

3. Srinivasan N, Oswal A, Garg S, Nahar J, Gosmonova A, Nahar R, et al. Cold agglutinin induced hemolysis in a newly diagnosed systemic lupus erythematosus. Am J Med Sci 2010;339:270-3.

4. Kotani T, Takeuchi T, Kawasaki Y, Hirano S, Tabushi Y, Kagitani M, et al. Successful treatment of cold agglutinin disease with anti-CD20 antibody (rituximab) in a patient with systemic lupus erythematosus. Lupus 2006;15:683-5.

5. Gupta N, Sharma S, Seth T, Mishra P, Mahapatra M, Kumar S, et al. Rituximab in steroid refractory autoimmune hemolytic anemia. Indian J Pediatr 2012;79:803-5.

6. Glaser M, Glaser A, Skalicky M. Long lasting remission by rituximab in a patient with primary cold agglutinin autoimmune hemolyticanemia. Wien Klin Wochenschr 2011;2013:680-3.

7. Peñalver FJ, Alvarez-Larrán A, Díez-Martin JL, Gallur L, Jarque I, Caballero D, et al. Rituximab is an effective and safe therapeutic alternative in adults with refractory and severe autoimmune hemolytic anemia. Ann Hematol 2010;89:1073-80. 